\title{
Demand Driven Evolution of the Cognitive Pilot Channel
}

\author{
Markus Mueck \\ Infineon Technologies AG \\ Am Campeon 1-12 \\ 85579 Neubiberg, GERMANY \\ Email:MarkusDominik.Mueck@infineon.com
}

\author{
Thomas Haustein \\ Fraunhofer Heinrich Hertz Institute \\ Broadband Mobile Communications Department \\ Einsteinufer 37, 10587 Berlin, GERMANY \\ Email: Thomas.Haustein@hhi.fraunhofer.de
}

\begin{abstract}
This contribution focuses on the context of massively heterogeneous wireless communication systems and details new enabling mechanisms for guaranteeing an efficient usage of the available resources: Building on the known concept of a Cognitive Pilot Channel (CPC), the user is enabled to obtain a User Class dependent Virtual Connectivity Map (VCM) and is thus able to build on a-priori knowledge of the surrounding wireless framework for ahead planning of optimum horizontal/vertical handovers. Furthermore, it is illustrated how statistical modeling is exploited for characterizing the typical behavior of various communication systems, for example in the TV White Spaces (TVWS); the latter approach enables a statistical evaluation of the expected reliability and longevity of a radio link operated as secondary user. Finally, an Incentive Based Distributed Sensing concept is introduced which introduces a context dependent rewarding system for motivating Mobile Devices (MD) to contribute to a collaborative radio context sensing effort.
\end{abstract}

Keywords- Cognitive Pilot Channel, Cognitive Radio (CR), ETSI, Software Defined Radio (SDR)

\section{INTRODUCTION}

The availability of heterogeneous Radio Access Technologies (RATs) at any given location, at any given time is already a reality today. Cellular systems, Wireless Local Area Networks (WLANs), etc. are quasi omnipresent in densely populated areas. A look at current standardization activities indicates that this evolution towards a heterogeneous radio framework will continue at least over the next few years: Cellular systems will evolve towards 3GPP Long Term Evolution (3GPP LTE) and then 3GPP Long Term Evolution Advanced (3GPP LTE-A), various new WiFi flavors are about to be introduced to the market such as high throughput WiFi (IEEE 802.11n), ultrahigh throughput WiFi (IEEE 802.11ac/ad), WiFi in TV White Spaces (IEEE 802.11af), etc. Furthermore, a new class of Metropolitan Area Networks is on the verge of hitting the market under the pseudonym WiMAX (IEEE $802.16 \mathrm{e} / \mathrm{m}$ ). Obviously, the efficient operation of such a diverse radio framework is a major challenge.

In recent years, various large-scale research projects studied the feasibility of efficient operation of a massively heterogeneous radio context, exploiting original ideas of [1]: IST-E ${ }^{2} \mathrm{R}$, IST-E ${ }^{2} \mathrm{R}$ II [2] and ICT-E ${ }^{3}$ [3] are some well-known examples.
As a result of the corresponding effort, it is stated that the global trend tends towards quickly rising data traffic in wireless networks while revenues for data services can't follow due to flat rate models putting tremendous pressure on the telecom industry to reduce CAPEX and OPEX. Approaches towards flexible spectrum usage and self organization of network components (SON) are currently taken from academic research into hot standardization topics to answer the mentioned challenges. As a consequence we observed a rising demand for cognitive radio environment knowledge towards the network and the terminal side.

It is assumed that the Self Organization principles will be extended in the future on both the Network (NW) and Mobile Device (MD) side in order to allow a cost- and latencyefficient operation of the network. A key issue here is the provision of suitable Context Information to MDs and their exploitation for collaborative Context Acquisition (or sensing) for having the required knowledge available on both the NW and the MD side. A first step into this direction has been introduced by the proposal of the Cognitive Pilot Channel (CPC) concept [4] and a corresponding overall heterogeneous system architecture approach [5].

In the context of this paper, we intend to enrich the existing state-of-the-art solutions by novel mechanisms in order to achieve an optimization of a multi-band and multi-RAT environment following metrics like throughput, QoS or minimization of handovers. So the key is efficient interaction between the base stations infrastructure and the user equipments at the other end. When discussing CPC issues, downlink coverage and resource efficiency are very important. We will consider two mayor approaches and combinations of them. In a network driven approach the wireless network can recommend or force policies towards the terminals. These radio access policies may be valid in general, for individual terminals, device groups or user groups. For a purely terminal driven approach, a novel Virtual Connectivity Map (VCM) based approach is proposed with the network providing load or coverage indicators for different radio access technologies (RAT); alternatively, terminals can request the distribution of such a Virtual Connectivity Map which is typically broadcasted on the CPC locally. The option of broadcasting (BC) or multicasting (MC) of the VCM strongly depends on the density of $\mathrm{BS}$, terminals and the demands for $\mathrm{VCM}$ 
provisioning. The VCM contents are designed to depend on MD User Classes, taking for example mobility parameters, RAT support capabilities, etc. into account. The concerned MDs will only receive and use those VCMs that provide useful information for its specific needs. For example, the information will typically differ for static users which might connect to pico / femto cells or WiFi access points (AP) or for high velocity users which are better catered for in larger cells with LTE or WiMAX. Furthermore, we have to consider the different types of wireless networks in an area, which might be open networks, closed subscriber groups or network with specific access condition e.g. a certain time or a price paid per use or willingness to report sensing data about the radio environment observed by the terminal.

In order to discuss the aspects of data base collection and distribution, CR decision making in more detail we will organize the paper as follows: Section II will focus on CR decisions based on long-term and short-term statistical traffic data collection, decision making and self learning. Section III discusses the CPC data base approach and how this information can be provided using a CPC approach. This is further detailed by introducing a distributed radio environment sensing using multi-band terminals for long-term and shortterm feedback to the network. Section IV details the actual data distribution via the CPC and Section V will finally summarize and conclude our discussions.

\section{COGNitive Radio Decision MaKing BASED ON STATISTICAL DATA AND OBTAINMENT OF STATISTICAL DATA}

A CPC concept is typically suitable in order to provide statistical data representing a large-scale and long-term statistical behavior of the system. For a user intending to operate its MD at a given time and given location, those longterm statistics, however, are of limited usefulness; the issue lies in the fact that they do not contain reliable data for ensuring the expected Quality of Service (QoS) at the given moment for a single user. Also, it is quasi-impossible to include local, heavily time-dependent statistics into a global database whose content is distributed by a CPC; the content is likely to be outdated once it is collected, verified, suitably reformatted and again distributed. It is thus proposed that MDs build on a combination of long- and short-term statistics. Long-term statistics, such as availability of specific RATs, average QoS measures, availability of White Spaces, etc. are expected to be provided by the CPC. Short-term statistics, such as current load-characteristics of specific RATs (e.g., is the given WiFi link constantly overloaded at the given time?, etc.) will be dealt with locally within the MDs; alternatively, a collaborative sensing approach can be applied using peer-topeer information exchange between the MDs.

A suitable way for modeling the behavior of RATs in a given (long-term) time-frame is proposed to be based on a Markov model as illustrated in Fig. 1 and Fig. 2; in these examples, the variables $a_{n}, b_{n}, d_{n}$ represent the transition probabilities for a typical discrete time birth-death Markov model.

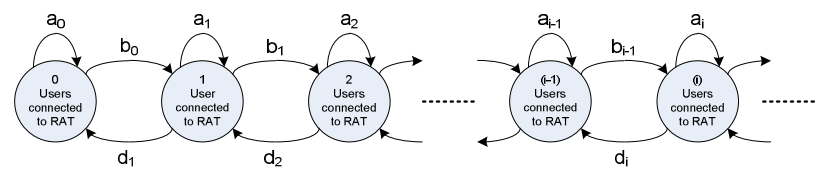

Figure 1: Proposal of a RAT State Diagram.

While Fig. 1 presents the rather generic case of measuring the arrival and departure probabilities of users, Fig. 2 follows a quite practical line by measuring saturation states based on a simplistic model consisting of the state set \{Small Number of Users, Medium Number of Users, Large Number of Users, Too Many Users\}.

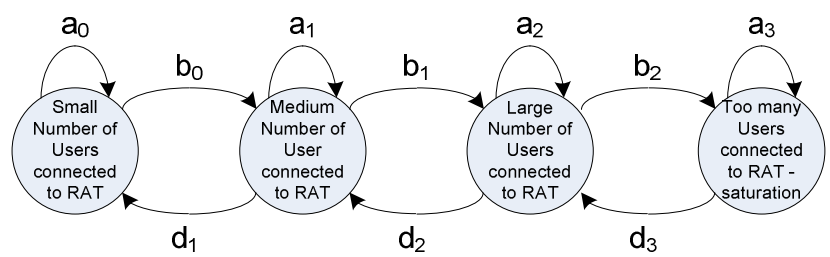

Figure 2: Proposal of a RAT State Diagram Representation - instead of a number of user, the more abstract states "small number of users", "medium number of users", "large number of users" and "too many users (saturation)" are used.

The same approach is proposed to be applied in order to characterize the QoS of TVWS frequency bands. Even if the presence of primary users is, it is important for MDs to have knowledge about the usage characteristics of other secondary users that may be present (locally, at a given time) in order to evaluate the expected QoS of a TVWS link.

Finally, the CPC based long-term statistics are communicated either by broadcasting the Markov state transition probabilities or the resulting evaluation of the probability that the system will be in a given state within a pre-defined timeframe. These are combined with the short term observations. The latter approach in particular enables a MD in order to evaluate the probability of having an immediate saturation of a given radio link.

\section{CPC DATA-BASE APPROACH AND DISTRIBUTED SENSING}

The Federal Communications Commission (FCC) has recently defined a set of rules to be met for any type of devices intending to use the TVWS as secondary user [6]. In particular, the combination of a centralized data-base with a local sensing approach is detailed. The general idea is based on the observation that local sensing alone is not sufficient in order to protect primary users (due to shadowing effects, inefficient sensing algorithms, etc. the misdetection probability is expected to be too high). 


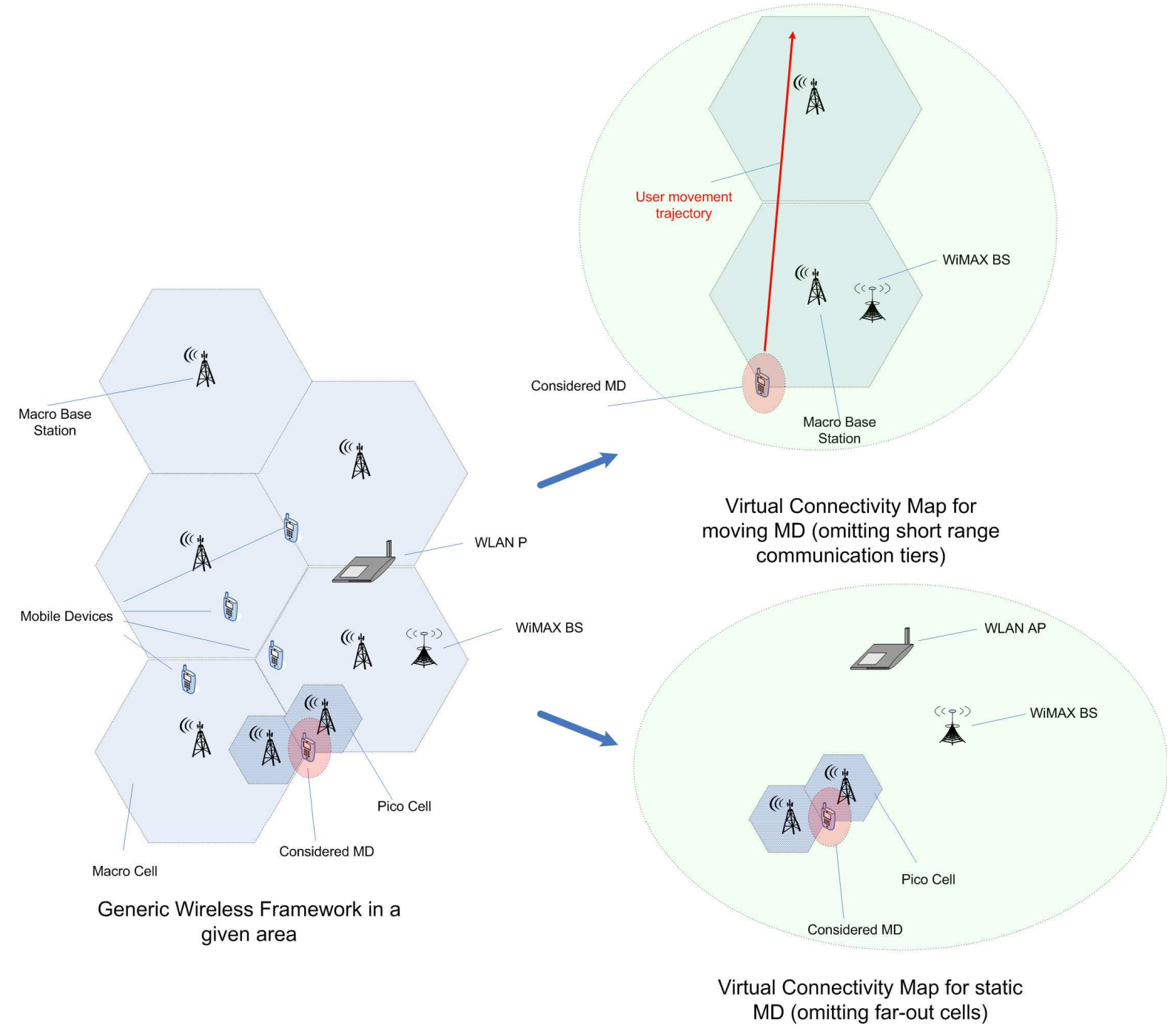

Figure 3: User Class dependent Virtual Connectivity Map.

Consequently, future users of TVWS bands need to consult a centralized data-base which gathers knowledge on the intended usage of the band by a primary system. Only if this data-base entry suggests that the band is free for usage by secondary users, a final sensing based check is performed and finally the MDs may apply opportunistic spectrum usage in the concerned bands.

In the framework of this paper, it is suggested that such a centralized data-base will also trigger (distributed) sensing within MDs in order to obtain further information of the radio context. This information is then broadcasted, typically via a $\mathrm{CPC}$, in order to further support the decision making processes in the MDs. Modern laptops and smart phones have sometimes 3 or more air-interfaces included e.g. GPRS, WiFi, HSPA or WiMAX. Adding geographical locations or relative positions to several known access points, e.g. in a cellular network allows to build a location based Virtual Connectivity Map (VCM). As it is illustrated in Fig. 3, the VCM of importance to a given device depends on its current User Class, taking in particular into account mobility characterustics (such as Mobility User Class $\in$ \{Static User, Low Mobility User, High Mobility User\}), RAT support characterustics (such as RAT Support User Class $\in\{3 G P P$ UMTS, 3GPP LTE, WiFi, WiMAX, ... \}), multi-mode support (such as Multi-Mode Support User Class $\in\{3 G P P$ UMTS and WiFi can be operated simultaneously, WiMAX and WiFi can be operated simultaneously, ...\}), etc. The corresponding VCM elements of importance to the considered MD taking the Mobility User Class into account are highlighted in Fig. 3: The moving MD's VCM contains information of Macro Cells and Metropolitan Area Networks, but omits Pico-Cells and short range communication systems; on the other hand, the static MD's VCM focuses on local Pico Cells and gives furthermore information on available short range and Metropolitan Area Network systems. 
We can expect repeated pattern of user terminal distribution and user communication activity which allow optimization of the wireless network at certain time instances on the one hand and wireless network load prediction on the other hand. This information can be distributed to all access points within the area of the VCM e.g. for self optimization of their radio resources and can be provided to the user terminals helping them to reduce effort for first hand spectrum sensing when entering a new area. The VCM information can be provided to the users in different ways: First, it can be broadcasted in a distributed CPC manner as described in the section before using all access point technologies or, secondly provided individually to the users on demand. The rationale behind the second approach is that many terminals which can be incorporated into the distributed sensing process might not be able to communicate over multiple radio access technologies and therefore precise information of the VCM is of more value for multi standard terminals which can request a VCM report on demand. State of the art multi-standard terminals go for best connection according to predefined policies or require manual settings by the user himself both are sub-optimum for an efficient use of the available spectrum and are based on instantaneous and individual decisions by each terminal alone without benefiting from the network intelligence e.g. suggestions for access technologies and connection policies. Furthermore, this VCM information is very important for cognitive communication systems which either operate sporadically or newly enter the coverage area.

In order to benefit from the concept discussed above we have to make sure that, first, the terminal can obtain and provide the necessary sensing information which requires deeper access to PHY and MAC layer parameters from a higher layer multistandard radio sensing entity and a predefined, preferably standardized feedback sensing data reporting channel which might be easily manageable as an over-the-top solution at IPlevel. The second crucial point is the willingness of users to perform sensing and to provide this information to the network. Here, issues like battery draining and privacy issues can influence the user to be non-cooperative. Therefore we consider measures for stimulating the user's willingness very essential to make Distributed and Collaborative Sensing work in business practice.

The issue of privacy can be solved when the over the top application assures anonymity for the user so he can be sure that his sensing feedback will not be used for sophisticated user tracking.

A possible approach can be Incentive Based Sensing Request by the network. A single or a plurality of wireless network operators might offer incentives like e.g. free roaming minutes or free SMS when users are willing to report radio sensing information. When enough users are available, the network can request sensing reports in regular intervals giving the option to terminals only to report if observed radio environment conditions change. This makes sure that terminals don't drain their battery when being stationary for instances while the owner is sleeping. At the same time the terminals can report on ramp up and shut-down activity of localized networks nodes like femtos or WiFi APs. The later point can also be considered as an enabling technique to optimize e.g. WiFi frequency band coordination ( $\mathrm{SON}$ ) locally and dynamically based on automated sensing techniques. Going one step further, the network can collect at least radio wise an AP-owner/operator independent central VCM which might allow to activate femtos and WiFi AP on demand if members of the closed subscriber group (CSG) report their position inside the VCM and request wake-up on IP over e.g. the DSL line triggered by a service in the internet. This allows longer shut down times of radio access points with limited users having access and therefore helps to reduce power consumption and frequency occupation by idle APs. In this way reliability and QoS can be improved even for LAN equipment sharing load information dynamically.

\section{COGNitive Pilot ChanNEL AND DistribUtion OF INFORMATION FROM THE NETWORK}

Having collected radio connectivity parameters data bases and distributed sensing in heterogeneous wireless networks the question arises which conclusions, rules, policies can be drawn from these enormous amount of date and as a next step how to make the resulting information e.g. decision rules, access policies or VCM accessible to terminals incorporating varying multi-standard capabilities. We propose a multidimensional hierarchical distribution of this information over a $C P C$ characterized briefly in the following.

The CPC defines channels to distribute required multi-band information to terminals in a wireless network in order to facilitate the operations of cognitive radio access. The CPC provides information on which air-interfaces can be expected at certain locations and time. This information may include operator information, RAT type and allocated frequency bands. Exemplary scenarios where the CPC is seen beneficial are:

a) The $\mathrm{CPC}$ is used to support terminals during the start-up phase in an unknown or changing radio environment.

b) In the context of a secondary frequency use, CPC can facilitate the exchange of spectrum sensing information between terminals and a serving base station in order to perform collaborative/cooperative sensing the search and monitoring of white spaces to start communication.

c) The CPC can be used for an efficient level of collaboration between a network and the terminals by supporting Radio Resource Management (RRM) optimization procedures.

\section{Hierarchical Cognitive Pilot Channel Design:}

Taking the multitude of options for MD classes into account it becomes obvious that different $\mathrm{MD}$ classes might require different CPC information e.g. VCM or inter-RAT hand-over policies. In order to avoid unnecessary signaling overhead in multiple CPCs we propose the following hierarchical CPC design.

The cellular network e.g. WiMAX is considered to broadcast a basic CPC containing general information about available wireless networks in licensed spectrum and where (e.g. 
frequency band and RAT) further information on private wireless networks (e.g. in ISM bands) can be obtained either in repeated regular broadcasts or on demand.

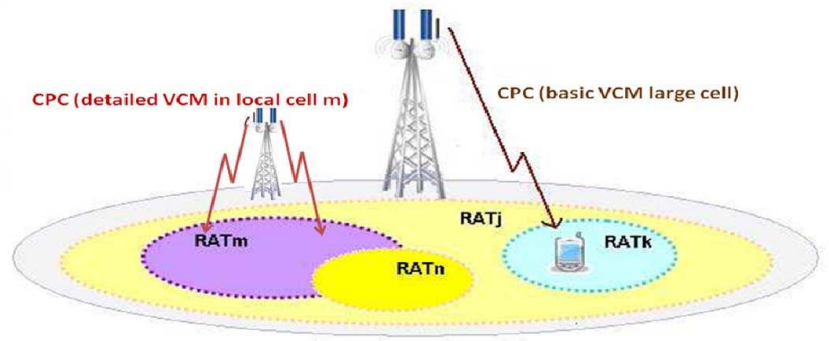

Figure 4: Hierarchical CPC design in heterogeneous wireless networks

By these means CPC overhead can be balanced with the repetition rate of more detailed $\mathrm{CPC}$ information in a hierarchical manner. After receiving the basic CPC, each MD can make individual decisions for deeper CPC extraction or demanding for specific CPC information. The hierarchy can follow various criteria like cellular range, mobility classes, RAT types or access restriction categories.

This concept naturally incorporates an overlay of centralized outband CPC and localized inband CPCs in a scalable fashion, allowing heterogeneous networks to be included in the overall concept of cognitive network information distribution such that embedded CPC broadcasting solutions and over the top CPC multicast and uni-cast solutions (e.g. pure IP based) can be supported at the same time. From a standardization point this allows upgrading existing wireless systems such that they can be included in a hierarchical CPC design by signaling their specific kind of CPC provisioning over standardized CPCs of more advanced or well established wireless systems.

Here again, further refinement of details in CPC information can be optimized by opting e.g. between broad-cast, multi-cast and uni-cast of information like a detailed VCM. Simply following individual user requests on VCM distribution using uni-cast only can blow up overhead tremendously, therefore each access point can distribute its CPC information again in a hierarchical manner e.g. sending most commonly requested information in regular broadcast, followed by multi-casting MD class specific information after MDs have registered to the wireless network. In depth knowledge of the VCM can be provided on demand or broadcasted in long intervals of the order of minutes or hours allowing MDs to learn RAT and spectrum options and policies about their radio environment e.g. overnight and benefit next morning when communication traffic rises again.

In return the CPC can be used to trigger distributed sensing reports obtained by specific MD classes in a localized area. A kind of collaborative sensing can be established allowing the network to measure network optimization procedures like downlink power control, fractional frequency reuse or beamsteering/ antenna tilting in a closed loop response system at very low delay and with quasi static measurement nodes. This technique allows dynamic pro-active cell capacity optimization when e.g. many users are inside a building at office hours with many MDs just in idle mode before foreseeable events like lunch breaks will increase data and voice traffic significantly.

Especially, if it comes e.g. to ad-hoc white space communication the many options of access to CPCs and VCM make decisions of white space choices and knowledge about local usage constraints more reliable since the hidden terminal problem cannot be combated by distributed sensing itself if e.g. only two nodes are present. Probabilities of primary or secondary white space occupancy can be provided from the network supporting new MDs in choosing their preferred white spaces accordingly. Furthermore, especially CPC request channels can further be used to temporarily register spectrum usage including location, power, bandwidth and expected temporal behavior. This information can be shared among MDs looped back over the local CPC allowing efficient autonomous spectrum coordination using game theoretic approaches or predefined policies recommended or enforced by the network or spectrum authorities.

\section{CONCLUSIONS}

With a focus on massively heterogeneous radio systems, known context provisioning solutions, namely the Cognitive Pilot Channel concept, have been extended. In particular, the importance of combining long-term and short-term statistics has been illustrated and corresponding Markov model based practical solutions have been outlined. Furthermore, the advantages of introducing a User Class dependent Virtual Connectivity Map (VCM) as well as an novel Incentive Based Sensing Request approach have been detailed and explained. The next steps consist in advocating these approaches in relevant standardization bodies, such as ETSI RRS, 3GPP, etc. and to further detail inherent protocols and information flows.

\section{REFERENCES}

[1] J. Mitola III, Cognitive Radio, Ph.D. thesis, KTH, Stockholm, Sweden, 2000

[2] FP6 E2R Programme Achievements and Impact, Dr. Didier Bourse, Dr. Markus Muck, Dr. David Bateman, Dr. Soodesh Buljore, Dr. Nancy Alonistioti, Dr. Klaus Moessner, Mr. Eric Nicollet, Dr. Enrico Buracchini, Pr. Panagiotis Demestichas, Makis Stamatelatos, Eleni Patouni, Proceeding of the SDR 07 Technical Conference and Product Exposition, available at

http://www.sdrforum.org/pages/sdr07/Proceedings/Papers/Invited/12.5001 invitedPaper1_Bourse.pdf

[3] ICT-2007-216248 E3 Project, http://www.ict-e3.eu/

[4] ETSI TR 102 683: "Reconfigurable Radio Systems (RRS); Cognitive Pilot Channel (CPC)", 2009

[5] IEEE Standard 1900.4 for Architectural Building Blocks Enabling Network-Device Distributed Decision Making for Optimized Radio Resource Usage in Heterogeneous Wireless Access Networks, Feb. 27, 2009

[6] http://www.fcc.gov/oet/cognitiveradio/ 